\title{
Kink oscillations of cooling coronal loops with variable cross-section
}

\author{
M. S. Ruderman ${ }^{1,2}$, A. A. Shukhobodskiy ${ }^{1,3}$, and R. Erdélyi ${ }^{1,4,5}$ \\ ${ }^{1}$ Solar Physics and Space Plasma Research Centre ( $\left.\mathrm{SP}^{2} \mathrm{RC}\right)$, University of Sheffield, Hicks Building, Hounsfield Road, \\ Sheffield S3 7RH, UK \\ e-mail: m.s.ruderman@sheffield.ac.uk \\ 2 Space Research Institute (IKI) Russian Academy of Sciences, 117342 Moscow, Russia \\ 3 ITMO University, Kronverkskii ave 49, 197101 Saint-Petersburg, Russia \\ ${ }^{4}$ Debrecen Heliophysical Observatory (DHO), Research Centre for Astronomy and Earth Sciences, Hungarian Academy of Sciences, \\ 4010 Debrecen, PO Box 30, Hungary \\ 5 Key Laboratory of Solar Activity, National Astronomical Observatories, Chinese Academy of Sciences, 100012 Beijing, PR China \\ Received 29 November 2016 / Accepted 6 March 2017
}

\begin{abstract}
We study kink waves and oscillations in a thin expanding magnetic tube in the presence of flow. The tube consists of a core region and a thin transitional region at the tube boundary. In this region the plasma density monotonically decreases from its value in the core region to the value outside the tube. Both the plasma density and velocity of background flow vary along the tube and in time. Using the multiscale expansions we derive the system of two equations describing the kink oscillations. When there is no transitional layer the oscillations are described by the first of these two equations. We use this equation to study the effect of plasma density variation with time on kink oscillations of an expanding tube with a sharp boundary. We assume that the characteristic time of the density variation is much greater than the characteristic time of kink oscillations. Then we use the Wentzel-Kramer-Brillouin (WKB) method to derive the expression for the adiabatic invariant, which is the quantity that is conserved when the plasma density varies. The general theoretical results are applied to the kink oscillations of coronal magnetic loops. We consider an expanding loop with the half-circle shape and assume that the plasma temperature inside a loop decays exponentially with time. We numerically calculated the dependences of the fundamental mode frequency, the ratio of frequencies of the first overtone and fundamental mode, and the oscillation amplitude on time. We obtained that the oscillation frequency and amplitude increase and the frequency ratio decreases due to cooling. The amplitude increase is stronger for loops with a greater expansion factor. This effect is also more pronounced for higher loops. However, it is fairly moderate even for loops that are quite high.
\end{abstract}

Key words. magnetohydrodynamics (MHD) - plasmas - waves - Sun: corona - Sun: oscillations

\section{Introduction}

After transverse oscillations of coronal magnetic loops were first observed by the Transition Region and Coronal Explorer (TRACE) spacecraft and reported by Aschwanden et al. (1999) and Nakariakov et al. (1999), they received ample attention from solar physicists. These oscillations have been interpreted as fast standing kink body waves in cylindrical magnetic flux tubes. In the earlier studies dealing with the interpretation of transverse oscillations, the simplest model of a coronal loop was used: a straight homogeneous magnetic flux tube (e.g. Ryutov \& Ryutova 1976; Edwin \& Roberts 1983). Later, more complex and realistic models of coronal loops were developed. For a review of theory of transverse coronal loop oscillations see e.g. Ruderman \& Erdélyi (2009).

One particularly important property of coronal loops is the plasma density variation along the loop. Andries et al. (2005b) showed that this effect leads to the reduction of the ratio of the fundamental mode to the first overtone frequency below the canonical value of 2 of a homogeneous loop. On the basis of this result, Andries et al. (2005a) suggested the method of estimation of the atmospheric scale height in the solar corona, using the frequency ratio (see also McEwan et al. 2008, or for a review Andries et al. 2009). Dymova \& Ruderman (2005) showed that kink oscillations of thin magnetic tubes with the density varying along the tube can be described by the wave equation with the phase speed equal to the local kink speed. Although typically the coronal loop expansion is relatively small, the ratio of the loop cross-section radii at the apex and at the footpoints can still reach a value of 1.5 (Watko \& Klimchuk 2000; Klimchuk et al. 2000). It is surprising that in the chromosphere the expansion of vertical magnetic flux tubes can reach a value of up to a few hundred (e.g. Tsuneta et al. 2008). Ruderman et al. (2008) and Verth \& Erdélyi (2008) generalised the derivation by Dymova \& Ruderman (2005) for the case where the density and cross-section radius both vary along the loop. Using the wave equation that they derived, they showed that the two effects act in the opposite direction. While the density variation causes the reduction of the frequency ratio, the tube expansion causes its increase. Interestingly, these inhomogeneity effects show similar behaviour for sausage oscillations (Luna-Cardozo et al. 2012).

Observations by the Solar and Heliospheric Observatory (SoHO; Brekke et al. 1997; Winebarger et al. 2002), TRACE (Winebarger et al. 2001) and Hinode (Chae et al. 2008; Ofman \& Wang 2008; Terradas et al. 2008) showed that flows are ubiquitously present in active region loops. Hence, it is important to study how flows in coronal loops modify the properties of kink oscillations. Gruszecki et al. (2008) have studied 
numerically the kink oscillations of coronal loops in the presence of field-aligned flow using the slab model. Terradas et al. (2010) studied the effect of flow on the period and damping rate of propagating kink oscillations in a magnetic tube homogeneous in the longitudinal direction. Erdélyi et al. (2014) studied how a "jet", i.e. a flow pulse, would affect the eigenoscillations as a jet propagates along the waveguide from one end to another. Ruderman (2010) derived the equation describing the kink oscillations of a magnetic tube with flow in the thin tube approximation. He assumed that the tube cross-section radius is constant; however, the density and flow velocity can vary both along the tube and in time. Using this equation he studied the effect of flow on the eigenfrequencies of kink oscillations.

It is very often observed that oscillating coronal loops are in a highly dynamic state. In particular, they can cool quickly with a characteristic cooling time of the order of a few periods of the kink oscillation (e.g. Aschwanden \& Terradas 2008; Aschwanden \& Schrijver 2011). This cooling can strongly affect the kink oscillations. The kink oscillations of cooling coronal loops have been studied by Morton \& Erdélyi (2009, 2010). In particular, Morton \& Erdélyi (2009) found that cooling causes the decrease in the period of kink oscillations. Ruderman (2011b) used the equation derived by Ruderman (2010) to study the kink oscillations of a coronal loop with the density varying along the loop and in time. In the approximation of slow temporal variation he derived the expression for an adiabatic invariant, which is a quantity conserved during the density evolution. Using this invariant he showed that cooling causes the amplification of kink oscillations. Similar results were obtained for longitudinal oscillations (see Al-Ghafri \& Erdélyi 2013; Al-Ghafri et al. 2014).

Ruderman (2010) assumed that the density can vary along the loop, but it does not vary in the transverse direction. Ruderman (2011a) generalised this study to also include the density variation in the transverse direction from a larger value inside the loop to a smaller value in the surrounding plasma. He derived the system of equations describing the kink oscillations of a loop with such a density transition and studied the kink oscillations of cooling loops damped due to resonant absorption. Recently the kink oscillations of radiatively cooling coronal loops have been studied numerically by Magyar et al. (2015).

In this article, we generalise the studies by Dymova \& Ruderman (2005), Ruderman et al. (2008), and Ruderman (2010, 2011a,b), and derive the system of equations describing the kink oscillations of an expanding loop with the density varying both along and across the loop, as well as in time, and in the presence of background flow. We then use this system to study the effect of cooling on kink oscillations of an expanding magnetic loop.

The paper is organised as follows. In the next section we describe the equilibrium state and present the governing equations. In Sect. 3 we transform the linearised MHD equations. In particular, we introduce the plasma displacement. In Sect. 4 we derive the governing equation for the kink oscillations in the thin tube approximation. In Sect. 5 we present the general analysis of the eigenvalue problem describing the kink oscillations in the presence of a stationary background flow. In Sect. 6 we derive the expression for the adiabatic invariant for the kink oscillations of an expanding magnetic tube with the slowly varying density. We then use this invariant to study the effect of cooling on the coronal loop kink oscillations. Section 7 contains the summary of the obtained results and our conclusions.

\section{Equilibrium state and governing equations}

We model a coronal loop as a straight, thin, and expanding magnetic tube with a circular cross-section. The tube can be either finite or semi-infinite, so that we can consider both standing and propagating waves. The tube consists of a core and a transition region where the density decreases from a higher value inside the tube to the lower value representing the surrounding plasma. In cylindrical coordinates $r, \phi, z$ with the $z$-axis coinciding with the tube axis, the plasma density is defined by

$\rho=\left\{\begin{array}{l}\rho_{i}(t, r, z), \quad 0 \leq r \leq R(z)(1-l / 2), \\ \rho_{t}(t, r, z), \quad R(z)(1-l / 2) \leq r \leq R(z)(1+l / 2), \\ \rho_{e}(t, r, z), \quad r \geq R(z)(1+l / 2),\end{array}\right.$

where $l$ is a constant determining the thickness of a transitional layer, $\rho(t, r, z)$ is a continuous function, and $\rho_{t}(t, r, z)$ is a monotonically decreasing function of $r$. We explain below why we split the tube into the core and transition layer. The equilibrium magnetic field is $\boldsymbol{B}=\left(B_{r}(r, z), 0, B_{z}(r, z)\right)$. The divergence-free condition for the magnetic field is written as

$\frac{1}{r} \frac{\partial\left(r B_{r}\right)}{\partial r}+\frac{\partial B_{z}}{\partial z}=0$

It follows from this equation that $\boldsymbol{B}$ can be expressed in terms of the magnetic flux function $\psi$ as

$B_{r}=-\frac{1}{r} \frac{\partial \psi}{\partial z}, \quad B_{z}=\frac{1}{r} \frac{\partial \psi}{\partial r}$.

We assume that the boundaries of the transitional layer are magnetic surfaces. This implies that the equations $r=R(z)(1-l / 2)$ and $r=R(z)(1+l / 2)$ can be written as $\psi=\psi_{i}=$ const. and $\psi=\psi_{e}=$ const., respectively, where $i$ and $e$ refer to internal and external values.

In what follows we use the cold plasma approximation. Then, to satisfy the equilibrium condition, the magnetic field must be potential. This implies that $\boldsymbol{B}$ must satisfy the equation

$\frac{\partial B_{r}}{\partial z}=\frac{\partial B_{z}}{\partial r}$

which can be rewritten in terms of the magnetic flux function as

$r \frac{\partial}{\partial r}\left(\frac{1}{r} \frac{\partial \psi}{\partial r}\right)+\frac{\partial^{2} \psi}{\partial z^{2}}=0$

There is a time-dependent background flow with the velocity $\boldsymbol{U}=\left(U_{r}(t, r, z), 0, U_{z}(t, r, z)\right)$. The flow velocity is assumed to be parallel to the equilibrium magnetic field, $\boldsymbol{U} \| \boldsymbol{B}$. The plasma density and velocity are related by the mass conservation equation

$\frac{\partial \rho}{\partial t}+\frac{1}{r} \frac{\partial\left(r \rho U_{r}\right)}{\partial r}+\frac{\partial\left(\rho U_{z}\right)}{\partial z}=0$.

The perturbations of the magnetic field and plasma velocity, $\boldsymbol{b}=$ $\left(b_{r}, b_{\phi}, b_{z}\right)$ and $\boldsymbol{u}=\left(u_{r}, u_{\phi}, u_{z}\right)$, are described by the linearised MHD equations in the cold plasma approximation,

$$
\begin{aligned}
& \frac{\partial \boldsymbol{u}}{\partial t}+(\boldsymbol{U} \cdot \nabla) \boldsymbol{u}+(\boldsymbol{u} \cdot \nabla) \boldsymbol{U}=\frac{1}{\mu_{0} \rho}(\nabla \times \boldsymbol{b}) \times \boldsymbol{B}, \\
& \frac{\partial \boldsymbol{b}}{\partial t}=\nabla \times(\boldsymbol{u} \times \boldsymbol{B}+\boldsymbol{U} \times \boldsymbol{b}), \\
& \nabla \cdot \boldsymbol{b}=0
\end{aligned}
$$

where $\mu_{0}$ is the magnetic permeability of free space. 


\section{Transformation of the governing equations}

To derive the governing equations for kink oscillations we follow Ruderman et al. (2008). However now there are two features that make the derivation more complex. The first is the presence of the transitional layer between the tube core and the external plasma. The crucial step in the derivation of the governing equation for kink oscillations of a tube with a sharp boundary is to use two conditions at the tube boundary: the continuity of the plasma displacement in the direction normal to the boundary and the magnetic pressure perturbation. Here we introduce the jumps of these quantities across the transitional layer. After that the derivation of the governing equation is the same as in the case of a sharp boundary. The second feature is the presence of flow. In our derivation we need a closed system of equations for the plasma displacement and magnetic pressure perturbation. To obtain this system the perturbations of the plasma velocity and magnetic field need to be expressed in terms of the plasma displacement. When there is no background flow these expressions are trivial. It is still possible to obtain these expressions even when there is background flow. However, its presence makes them quite cumbersome. The aim of this section is to derive these expressions and then to obtain a closed system of equations for the plasma displacement and magnetic pressure perturbation. Hence, the main result of this section is the system of Eqs. (38)-(42). 1961):

Below we use the following identities (e.g. Korn \& Korn

$$
\begin{aligned}
& 2(\boldsymbol{G} \cdot \nabla) \boldsymbol{F}=\nabla \times(\boldsymbol{F} \times \boldsymbol{G})+\nabla(\boldsymbol{F} \cdot \boldsymbol{G})-\boldsymbol{F}(\nabla \cdot \boldsymbol{G}) \\
&+\boldsymbol{G}(\nabla \cdot \boldsymbol{F})-\boldsymbol{F} \times(\nabla \times \boldsymbol{G})-\boldsymbol{G} \times(\nabla \times \boldsymbol{F}), \\
& \begin{aligned}
\nabla \cdot(\boldsymbol{F} \times \boldsymbol{G})= & \boldsymbol{G} \cdot(\nabla \times \boldsymbol{F})-\boldsymbol{F} \cdot(\nabla \times \boldsymbol{G}), \\
\nabla \times(\boldsymbol{F} \times \boldsymbol{G})= & (\boldsymbol{G} \cdot \nabla) \boldsymbol{F}-(\boldsymbol{F} \cdot \nabla) \boldsymbol{G} \\
& +\boldsymbol{F}(\nabla \cdot \boldsymbol{G})-\boldsymbol{G}(\nabla \cdot \boldsymbol{F}),
\end{aligned}
\end{aligned}
$$

where $\boldsymbol{F}$ and $\boldsymbol{G}$ are arbitrary vector-functions.

We consider a Lagrangian particle with the initial position defined by vector $\boldsymbol{a}$. A current position of this particle is $\boldsymbol{x}(t, \boldsymbol{a})$, so $\boldsymbol{x}(0, \boldsymbol{a})=\boldsymbol{a}$. The trajectory of this particle is given by $\boldsymbol{x}=$ $\boldsymbol{x}(t, \boldsymbol{a})$. In the unperturbed flow it is $\boldsymbol{x}=\boldsymbol{x}_{0}(t, \boldsymbol{a})$. We introduce the plasma displacement $\boldsymbol{\xi}=\boldsymbol{x}-\boldsymbol{x}_{0}$. In what follows, we consider $\boldsymbol{\xi}$ as a function of Eulerian coordinates, meaning that it is a function of $t$ and $\boldsymbol{x}$. The particle velocity is given by

$\widetilde{\boldsymbol{U}}=\left.\frac{\partial \boldsymbol{x}}{\partial t}\right|_{\boldsymbol{a}}=\left.\frac{\partial \boldsymbol{x}_{0}}{\partial t}\right|_{\boldsymbol{a}}+\left.\frac{\partial \boldsymbol{\xi}}{\partial t}\right|_{\boldsymbol{x}}+(\widetilde{\boldsymbol{U}} \cdot \nabla) \boldsymbol{\xi}$

where the subscripts $\boldsymbol{a}$ and $\boldsymbol{x}$ indicate that the derivative with respect to $t$ is calculated at constant $\boldsymbol{a}$ and constant $\boldsymbol{x}$, respectively. Now, $\partial \boldsymbol{x}_{0} / \partial t=\boldsymbol{U}\left(t, \boldsymbol{x}_{0}\right)$. In the linear approximation we can substitute $\boldsymbol{U}\left(t, \boldsymbol{x}_{0}\right)$ for $\widetilde{\boldsymbol{U}}$ in the last term on the right-hand side of Eq. (11). Now, the Eulerian perturbation of the velocity is $\boldsymbol{u}(t, \boldsymbol{x})=\widetilde{\boldsymbol{U}}(t, \boldsymbol{x})-\boldsymbol{U}(t, \boldsymbol{x})$. Then, using the approximate relation $\boldsymbol{U}(t, \boldsymbol{x}) \approx \boldsymbol{U}\left(t, \boldsymbol{x}_{0}\right)+(\boldsymbol{\xi} \cdot \nabla) \boldsymbol{U}$ we obtain from Eq. (11) in the linear approximation

$\boldsymbol{u}=\frac{\partial \boldsymbol{\xi}}{\partial t}+(\boldsymbol{U} \cdot \nabla) \boldsymbol{\xi}-(\boldsymbol{\xi} \cdot \nabla) \boldsymbol{U}$

where here and below the time derivative is calculated at constant $\boldsymbol{x}$. Using the first identity in Eq. (10) we transform this expression to

$\boldsymbol{u}=\frac{\partial \boldsymbol{\xi}}{\partial t}+\nabla \times(\boldsymbol{\xi} \times \boldsymbol{U})-\boldsymbol{\xi}(\nabla \cdot \boldsymbol{U})+\boldsymbol{U}(\nabla \cdot \boldsymbol{\xi})$.
Now we assume that the velocity $\boldsymbol{U}$ is potential, $\nabla \times \boldsymbol{U}=0$. Then, again using the first identity in Eq. (10), we obtain

$(\boldsymbol{U} \cdot \nabla) \boldsymbol{u}+(\boldsymbol{u} \cdot \nabla) \boldsymbol{U}=\nabla\left(U u_{\|}\right)-\boldsymbol{U} \times(\nabla \times \boldsymbol{u})$,

where $u_{\|}=\boldsymbol{b}_{0} \cdot \boldsymbol{u}$ and $\boldsymbol{b}_{0}=\boldsymbol{B} / \boldsymbol{B}$ is the unit vector in the magnetic field direction. Substituting this result in Eq. (7) and taking the scalar product of the obtained equation with $\boldsymbol{b}_{0}$ yields

$\frac{\partial u_{\|}}{\partial t}+\boldsymbol{b}_{0} \cdot \nabla\left(\boldsymbol{U} u_{\|}\right)=0$

Assuming that $u_{\|}=0$ for $t=0$ we obtain from this equation that $u_{\|}=0$ for $t>0$. Now, taking the scalar product of Eq. (13) with $\boldsymbol{b}_{0}$ and using the second identity in Eq. (10) yields

$\frac{\partial \xi_{\|}}{\partial t}=\frac{1}{B} \nabla \cdot\left(B U \boldsymbol{\xi}_{1}\right)+\xi_{\|} \nabla \cdot \boldsymbol{U}-U \nabla \cdot \boldsymbol{\xi}$,

where $\xi_{\|}=\boldsymbol{b}_{0} \cdot \boldsymbol{\xi}$ and $\boldsymbol{\xi}_{1}=\boldsymbol{\xi}-\boldsymbol{\xi}_{\|} \boldsymbol{b}_{0}$.

With the aid of the third identity in Eq. (10) we obtain

$\nabla \times(\boldsymbol{\xi} \times \boldsymbol{B})=\boldsymbol{f}-\boldsymbol{B}(\nabla \cdot \boldsymbol{\xi})$,

where

$\boldsymbol{f}=(\boldsymbol{B} \cdot \nabla) \boldsymbol{\xi}-(\boldsymbol{\xi} \cdot \nabla) \boldsymbol{B}$.

Using Eq. (12) we transform Eq. (8) to

$\frac{\partial \boldsymbol{b}}{\partial t}=\nabla \times(\boldsymbol{U} \times \boldsymbol{b})-\nabla \times\left[\boldsymbol{B} \times\left(\frac{\partial \boldsymbol{\xi}}{\partial t}+(\boldsymbol{U} \cdot \nabla) \boldsymbol{\xi}-(\boldsymbol{\xi} \cdot \nabla) \boldsymbol{U}\right)\right]$.

With the aid of Eq. (17) we reduce this equation to

$$
\begin{array}{r}
\frac{\partial}{\partial t}[\boldsymbol{b}-\boldsymbol{f}+\boldsymbol{B}(\nabla \cdot \boldsymbol{\xi})]=\nabla \times[ \\
\left.U \boldsymbol{b}_{0} \times(\boldsymbol{b}-(\boldsymbol{B} \cdot \nabla) \boldsymbol{\xi})\right] \\
+\nabla \times[\boldsymbol{B} \times(\boldsymbol{\xi} \cdot \nabla) \boldsymbol{U}] .
\end{array}
$$

Then, using the identity

$(\boldsymbol{\xi} \cdot \nabla) \boldsymbol{U}=\boldsymbol{B}(\boldsymbol{\xi} \cdot \nabla) \frac{U}{B}+\frac{U}{B}(\boldsymbol{\xi} \cdot \nabla) \boldsymbol{B}$,

we finally arrive at

$\frac{\partial}{\partial t}[\boldsymbol{b}-\boldsymbol{f}+\boldsymbol{B}(\nabla \cdot \boldsymbol{\xi})]=\nabla \times\left\{U \boldsymbol{b}_{0} \times[\boldsymbol{b}-\boldsymbol{f}+\boldsymbol{B}(\nabla \cdot \boldsymbol{\xi})]\right\}$.

It follows from this equation that $\boldsymbol{b}-\boldsymbol{f}+\boldsymbol{B}(\nabla \cdot \boldsymbol{\xi})=0$ for $t>0$ if it is zero at $t=0$, which is assumed below. Hence, we obtain

$\boldsymbol{b}=(\boldsymbol{B} \cdot \nabla) \boldsymbol{\xi}-(\boldsymbol{\xi} \cdot \nabla) \boldsymbol{B}-\boldsymbol{B}(\nabla \cdot \boldsymbol{\xi})$.

Using the third identity in Eq. (10) and recalling that the magnetic field is divergent-free we can rewrite this expression as

$\boldsymbol{b}=\nabla \times(\boldsymbol{\xi} \times \boldsymbol{B})$.

We now introduce the magnetic pressure perturbation $P=\boldsymbol{B}$. $\boldsymbol{b} / \mu_{0}$. Taking the scalar product of Eq. (24) with $\boldsymbol{B}$, using the second identity in Eq. (10), and recalling that the equilibrium magnetic field is potential, we easily obtain

$P=-\frac{1}{\mu_{0}} \nabla \cdot\left(B^{2} \xi_{1}\right)$.

Substituting Eqs. (14) and (24) in Eq. (7), and taking into account that $u_{\|}=0$ yields

$\frac{\partial \boldsymbol{u}}{\partial t}=\boldsymbol{b}_{0} \times\left(U \nabla \times \boldsymbol{u}-\frac{B}{\mu_{0} \rho} \nabla \times \nabla \times(\boldsymbol{\xi} \times \boldsymbol{B})\right)$. 
Equations (13), (16), and (26) constitute the system of equations for $\boldsymbol{\xi}$.

Now we introduce the components of the displacement and velocity that are perpendicular to the magnetic field lines and in the plains containing the $z$-axis,

$\xi_{\perp}=\xi_{r} b_{0 z}-\xi_{z} b_{0 r} \quad u_{\perp}=u_{r} b_{0 z}-u_{z} b_{0 r}$.

Then, recalling that $\boldsymbol{B}$ is solenoidal and potential, we transform Eqs. (16) and (25) to

$$
\begin{aligned}
& \frac{\partial \xi_{\|}}{\partial t}=\frac{\xi_{\perp}}{B}\left(b_{0 z} \frac{\partial(B U)}{\partial r}-b_{0 r} \frac{\partial(B U)}{\partial z}\right)-U^{2}\left[b_{0 r} \frac{\partial}{\partial r}\left(\frac{\xi_{\|}}{U}\right)+b_{0 z} \frac{\partial}{\partial z}\left(\frac{\xi_{\|}}{U}\right)\right], \\
& P=-\frac{B}{\mu_{0}}\left(\frac{b_{0 z}}{r} \frac{\partial(r w)}{\partial r}+\frac{B}{r} \frac{\partial \xi_{\phi}}{\partial \phi}-b_{0 r} \frac{\partial w}{\partial z}\right),
\end{aligned}
$$

where $w=B \xi_{\perp}$. To obtain the expression for $\boldsymbol{u}$ in terms of $\boldsymbol{\xi}$ we use Eq. (13). First, we take the scalar product of this equation with the vector $\left(b_{0 z}, 0,-b_{0 r}\right)$ and use the third identity in Eq. (10) to obtain the expression for $u_{\perp}$. Then we take the $\phi$-component of Eq. (13) to obtain the expression for $u_{\phi}$. As a result we arrive at

$u_{\perp}=\frac{1}{B} \frac{\partial w}{\partial t}+\frac{U}{B}\left(\frac{b_{0 r}}{r} \frac{\partial(r w)}{\partial r}+b_{0 z} \frac{\partial w}{\partial z}\right)$,

$u_{\phi}=\frac{\partial \xi_{\phi}}{\partial t}+U\left[r b_{0 r} \frac{\partial}{\partial r}\left(\frac{\xi_{\phi}}{r}\right)+b_{0 z} \frac{\partial \xi_{\phi}}{\partial z}\right]$.

Now we take the scalar product of Eq. (26) with the vector $\left(b_{0 z}, 0,-b_{0 r}\right)$. Using the third identity in Eq. (10) yields

$$
\begin{aligned}
\frac{\partial u_{\perp}}{\partial t}+ & U\left[r B_{r} \frac{\partial}{\partial r}\left(\frac{u_{\perp}}{r B}\right)+B_{z} \frac{\partial}{\partial z}\left(\frac{u_{\perp}}{B}\right)\right]=\frac{B}{\mu_{0} \rho}\left[\frac{\partial^{2} w}{\partial z^{2}}\right. \\
+ & \left.\frac{\partial}{\partial r}\left(\frac{1}{r} \frac{\partial(r w)}{\partial r}\right)+B_{z} \frac{\partial}{\partial r}\left(\frac{1}{r} \frac{\partial \xi_{\phi}}{\partial \phi}\right)-\frac{B_{r}}{r} \frac{\partial^{2} \xi_{\phi}}{\partial \phi \partial z}\right] .
\end{aligned}
$$

Then we take the $\phi$-component of Eq. (26), and again use the third identity in Eq. (10) to obtain

$$
\begin{aligned}
\frac{\partial u_{\phi}}{\partial t} & +U\left(\frac{b_{0 r}}{r} \frac{\partial\left(r u_{\phi}\right)}{\partial r}+b_{0 z} \frac{\partial u_{\phi}}{\partial z}\right)=\frac{B}{\mu_{0} \rho}\left[\frac{B}{r^{2}} \frac{\partial^{2} \xi_{\phi}}{\partial \phi^{2}}\right. \\
& +\frac{b_{0 z}}{r^{2}} \frac{\partial^{2}(r w)}{\partial \phi \partial r}-\frac{b_{0 r}}{r} \frac{\partial^{2} w}{\partial \phi \partial z}+\frac{b_{0 r}}{r} \frac{\partial}{\partial r}\left(r^{2} B_{r} \frac{\partial}{\partial r}\left(\frac{\xi_{\phi}}{r}\right)\right. \\
& \left.\left.+r B_{z} \frac{\partial \xi_{\phi}}{\partial z}\right)+b_{0 z} \frac{\partial}{\partial z}\left(r B_{r} \frac{\partial}{\partial r}\left(\frac{\xi_{\phi}}{r}\right)+B_{z} \frac{\partial \xi_{\phi}}{\partial z}\right)\right] .
\end{aligned}
$$

Using Eq. (29) we reduce Eqs. (32) and (33) to

$$
\begin{aligned}
\frac{\partial u_{\perp}}{\partial t}+U\left[r B_{r} \frac{\partial}{\partial r}\left(\frac{u_{\perp}}{r B}\right)+B_{z} \frac{\partial}{\partial z}\left(\frac{u_{\perp}}{B}\right)\right]= \\
\frac{B^{2}}{\rho}\left[b_{0 r} \frac{\partial}{\partial z}\left(\frac{P}{B^{2}}\right)-b_{0 z} \frac{\partial}{\partial r}\left(\frac{P}{B^{2}}\right)\right] \\
+\frac{B^{2}}{\mu_{0} \rho}\left(r b_{0 r} \frac{\partial}{\partial r} \frac{1}{r}+b_{0 z} \frac{\partial}{\partial z}\right)\left(\frac{b_{0 r}}{r B} \frac{\partial(r w)}{\partial r}+\frac{b_{0 z}}{B} \frac{\partial w}{\partial z}\right), \\
\frac{\partial u_{\phi}}{\partial t}+U\left(\frac{b_{0 r}}{r} \frac{\partial\left(r u_{\phi}\right)}{\partial r}+b_{0 z} \frac{\partial u_{\phi}}{\partial z}\right)=-\frac{1}{\rho r} \frac{\partial P}{\partial \phi} \\
+\frac{1}{\mu_{0} \rho}\left(\frac{B_{r}}{r} \frac{\partial}{\partial r} r+B_{z} \frac{\partial}{\partial z}\right)\left(r B_{r} \frac{\partial}{\partial r}\left(\frac{\xi_{\phi}}{r}\right)+B_{z} \frac{\partial \xi_{\phi}}{\partial z}\right) .
\end{aligned}
$$

We see that Eq. (28) is detached from the other equations and only serves to determine $\xi_{\|}$. Hence we do not use it below. Equations (29)-(31), (34), and (35) constitute the system of equations for $\xi_{\perp}, \xi_{\phi}, u_{\perp}, u_{\phi}$, and $P$.

Now, following Ruderman et al. (2008), we use the magnetic flux function $\psi$ as an independent variable instead of $r$. With the aid of the chain rule we obtain the following relations:

$\frac{\partial f}{\partial r}=r B_{z} \frac{\partial f}{\partial \psi},\left.\quad \frac{\partial f}{\partial z}\right|_{r}=\left.\frac{\partial f}{\partial z}\right|_{\psi}-r B_{r} \frac{\partial f}{\partial \psi}$,

where $f$ is an arbitrary function, and the subscripts $r$ and $\psi$ indicate that the derivative is taken at constant $r$ and constant $\psi$, respectively. In particular, taking $f=r$ in these relations, we obtain

$\frac{\partial r}{\partial z}=\frac{B_{r}}{B_{z}}, \quad \frac{\partial r}{\partial \psi}=\frac{1}{r B_{z}}$

Using Eqs. (36) and (37) we transform Eqs. (29)-(31), (34), and (35) to

$$
\begin{aligned}
P= & -\frac{1}{\mu_{0}}\left(r B^{2} \frac{\partial w}{\partial \psi}+\frac{B^{2}}{r} \frac{\partial \xi_{\phi}}{\partial \phi}-B_{r} \frac{\partial w}{\partial z}+B_{z} \frac{w}{r}\right), \\
u_{\perp}= & \frac{1}{B} \frac{\partial w}{\partial t}+\frac{U_{z}}{r B} \frac{\partial(r w)}{\partial z}, \\
u_{\phi}= & \frac{\partial \xi_{\phi}}{\partial t}+r U_{z} \frac{\partial}{\partial z}\left(\frac{\xi_{\phi}}{r}\right), \\
\frac{\partial u_{\perp}}{\partial t}+ & r U B_{z} \frac{\partial}{\partial z}\left(\frac{u_{\perp}}{r B}\right)=\frac{r B B_{z}}{\mu_{0} \rho} \frac{\partial}{\partial z}\left(\frac{B_{z}}{r^{2} B^{2}} \frac{\partial(r w)}{\partial z}\right) \\
& +\frac{B^{2}}{\rho}\left[b_{0 r} \frac{\partial}{\partial z}\left(\frac{P}{B^{2}}\right)-r B \frac{\partial}{\partial \psi}\left(\frac{P}{B^{2}}\right)\right], \\
\frac{\partial u_{\phi}}{\partial t}+ & \frac{U_{z}}{r} \frac{\partial\left(r u_{\phi}\right)}{\partial z}=\frac{B_{z}}{\mu_{0} \rho r} \frac{\partial}{\partial z}\left[r^{2} B_{z} \frac{\partial}{\partial z}\left(\frac{\xi_{\phi}}{r}\right)\right]-\frac{1}{\rho r} \frac{\partial P}{\partial \phi} .
\end{aligned}
$$

The system of Eqs. (38)-(42) is used in the next section to derive the governing equations for the kink oscillations of a thin and expanding magnetic flux tube. Obviously, $u_{\perp}$ and $u_{\phi}$ can be eliminated using Eqs. (39) and (40). Hence, Eqs. (38)-(42) can be considered as a system of equations for $w=B \xi_{\perp}, \xi_{\phi}$, and $P$.

\section{Derivation of the governing equations}

We now assume that the magnetic tube is thin, $R(z) / L=O(\epsilon)$, where $\epsilon \ll 1$ and $L$ is the characteristic scale of variation of all equilibrium quantities, like the tube radius and magnetic field magnitude, along the tube. In the case of standing waves we can take $L$ equal to the tube length. We also assume that the characteristic scale of variation of perturbations along the tube is much larger than the tube radius. However, we do not make any assumption about the relation between $L$ and the characteristic scale of perturbation variation. In particular, they can be of the same order. In accordance with the assumption that the tube is thin we introduce the stretching variable along the tube defined as $Z=\epsilon z$. The characteristic time of the problem is $L / V_{*}$, where $V_{*}$ is the characteristic Alfvén speed. Its ratio to the Alfvénic time determined with respect to the tube radius, $R_{*} / V_{*}$, where $R_{*}$ is the characteristic value of the tube radius, is of the order of $\epsilon^{-1}$. Accordingly, we introduce the stretching time $T=\epsilon t$. The characteristic scale of variation of perturbations in the radial direction is $R_{*}$. However, we assume that the characteristic scale of variation of $\rho, B$, and $U$ outside of the transitional layer, i.e. in the core of the tube $(r<R(z)(1-l / 2))$ and outside of the tube 
$(r>R(z)(1+l / 2))$, is $L$. While the characteristic scale of variation of $B$ is $L$ even in the transitional layer, $\rho$ and $U$ can vary in this layer on the scale $l R_{*}$. The ratio of the radial and azimuthal component of the equilibrium magnetic field is of the order of $\epsilon$, and the same estimate is valid for the radial and azimuthal component of the equilibrium flow. In accordance with these estimates, we introduce the scaled radial components of the magnetic field and bulk flow velocity, namely $\widetilde{B}_{r}=\epsilon^{-1} B_{r}$ and $\widetilde{U}_{r}=\epsilon^{-1} U_{r}$.

From now on the derivation of governing equation is quite similar to that in Ruderman et al. (2008). It is based on using asymptotic expansions with respect to $\epsilon$. The main result of this section is Eq. (75) with the right-hand side given by Eq. (76).

Below we impose the restriction $B_{z}>0$. Since the tube is thin, in the vicinity of the magnetic tube we can approximate $\psi$ by the first term of its expansion in the Taylor series. Since the tube axis is a magnetic field line itself, $\psi=$ const. at $r=0$. Without loss of generality, we can take $\psi=0$ at $r=0$. Then it follows from Eq. (3) that the first term of the Taylor expansion of $\psi$ with respect to $r$ is proportional to $r^{2}$ and we can use the approximate expression

$\psi=\frac{1}{2} r^{2} h(z)$

Now it follows from Eq. (3) that the equilibrium magnetic field is given by

$B_{r}=-\frac{r}{2} h^{\prime}(z), \quad B_{z}=h(z)$,

where the prime indicates the derivative. For the magnetic field magnitude we obtain

$B=\sqrt{B_{r}^{2}+B_{z}^{2}}=B_{z}+O\left(\epsilon^{2}\right)=h(z)+O\left(\epsilon^{2}\right)$.

It follows from the magnetic flux conservation that

$h(z) R^{2}(z)=C=$ const.

For the flow speed we obtain

$U=\sqrt{U_{r}^{2}+U_{z}^{2}}=U_{z}+O\left(\epsilon^{2}\right)$

It follows from the symmetry that $\partial U / \partial r=0$ at $r=0$. Then, using Taylor's theorem, we obtain in the core region $(r<R(z)(1-l / 2))$

$$
\begin{aligned}
U(r, z) & =U(0, z)+\left.\frac{r^{2}}{2} \frac{\partial^{2} U}{\partial r^{2}}\right|_{r=r_{*}} \\
& =U(0, z)+O\left(R_{*}^{2} / L^{2}\right)=U(0, z)+O\left(\epsilon^{2}\right),
\end{aligned}
$$

where $0<r_{*}<R(z)(1-l / 2)$. Outside of the tube in its vicinity $(R(z)(1+l / 2)<r \ll L)$ we have

$$
\begin{aligned}
U(r, z) & =U(R(z)(1+l / 2), z)+\left.r \frac{\partial U}{\partial r}\right|_{r=r_{*}} \\
& =U(0, z)+O\left(R_{*} / L\right)=U(0, z)+O(\epsilon),
\end{aligned}
$$

where $R(z)(1+l / 2)<r_{*} \ll L$. Expressions similar to Eqs. (48) and (49) are also valid for $\rho$ outside of the transitional layer and for $B$ everywhere not far from the tube $(r \ll L)$. Hence, in the leading order approximation with respect to $\epsilon$, we can consider $U$ and $\rho$ as quantities independent of $r$ outside of the transitional layer, while $B$ can be considered as a quantity independent of $r$ everywhere in the region $r \ll L$.
We now integrate Eq. (6) over the area of the tube core crosssection, that is over a circle of radius $R(z)(1-l / 2)$. Below we assume that $l \ll 1$, meaning that one can neglect $l$ in comparison with unity. Then we obtain

$R^{2} \frac{\partial \rho}{\partial t}+R^{2} \frac{\partial\left(\rho U_{z}\right)}{\partial z}+\left.2\left(\rho R U_{r}\right)\right|_{r=R}=0$.

Since the velocity is parallel to the magnetic field, we have with the aid of Eqs. (44) and (46)

$\left.\frac{U_{r}}{U}\right|_{r=R}=\left.\frac{B_{r}}{B}\right|_{r=R}=-\frac{h^{\prime} R}{2 h}=R^{\prime}$.

Substituting this result in Eq. (50) and recalling that $B_{z} \approx B$, we eventually arrive at

$\frac{\partial \rho}{\partial t}+\frac{1}{R^{2}} \frac{\partial\left(\rho R^{2} U\right)}{\partial z}=0$

This equation is valid in the core of the tube.

In the new variables we transform Eqs. (38)-(42) to

$$
\begin{aligned}
& P=-\frac{1}{\mu_{0}}\left(r B^{2} \frac{\partial w}{\partial \psi}+\frac{B^{2}}{r} \frac{\partial \xi_{\phi}}{\partial \phi}-\epsilon^{2} \widetilde{B}_{r} \frac{\partial w}{\partial Z}+B_{z} \frac{w}{r}\right), \\
& u_{\perp}=\frac{\epsilon}{B} \frac{\partial w}{\partial T}+\frac{\epsilon U_{z}}{r B} \frac{\partial(r w)}{\partial Z}, \\
& u_{\phi}=\epsilon \frac{\partial \xi_{\phi}}{\partial T}+\epsilon r U_{z} \frac{\partial}{\partial Z}\left(\frac{\xi_{\phi}}{r}\right), \\
& \epsilon \frac{\partial u_{\perp}}{\partial T}+\epsilon r U B_{z} \frac{\partial}{\partial Z}\left(\frac{u_{\perp}}{r B}\right)=\epsilon^{2} \frac{r B B_{z}}{\mu_{0} \rho} \frac{\partial}{\partial Z}\left(\frac{B_{z}}{r^{2} B^{2}} \frac{\partial(r w)}{\partial Z}\right) \\
& \quad+\frac{B^{2}}{\rho}\left[\epsilon^{2} \tilde{b}_{0 r} \frac{\partial}{\partial Z}\left(\frac{P}{B^{2}}\right)-r B \frac{\partial}{\partial \psi}\left(\frac{P}{B^{2}}\right)\right], \\
& \epsilon \frac{\partial u_{\phi}}{\partial T}+\epsilon \frac{U_{z}}{r} \frac{\partial\left(r u_{\phi}\right)}{\partial Z}=\frac{\epsilon^{2} B_{z}}{\mu_{0} \rho r} \frac{\partial}{\partial Z}\left[r^{2} B_{z} \frac{\partial}{\partial Z}\left(\frac{\xi_{\phi}}{r}\right)\right]-\frac{1}{\rho r} \frac{\partial P}{\partial \phi}
\end{aligned}
$$

where $\tilde{b}_{0 r}=\epsilon^{-1} b_{0 r}$. Below we consider $\xi_{\perp}$ and $\xi_{\phi}$ as quantities of the order of unity. Then it follows from Eqs. (54) and (55) that $u_{\perp}=O(\epsilon)$ and $u_{\phi}=O(\epsilon)$. With the aid of these estimates we obtain from Eqs. (56) and (57) that $P=O\left(\epsilon^{2}\right)$. In accordance with these estimates we put $u_{\perp}=\epsilon \tilde{u}_{\perp}, u_{\phi}=\epsilon \tilde{u}_{\phi}$, and $P=\epsilon^{2} B^{2} Q$. Then, assuming that perturbations of all quantities are proportional to $\mathrm{e}^{\mathrm{i} \phi}$, we reduce the system of Eqs. (53)-(57) to

$$
\begin{aligned}
& r B^{2} \frac{\partial w}{\partial \psi}+\frac{\mathrm{i} B^{2} \xi_{\phi}}{r}+B_{z} \frac{w}{r}=O\left(\epsilon^{2}\right), \\
& \tilde{u}_{\perp}=\frac{1}{B} \frac{\partial w}{\partial T}+\frac{U_{z}}{r B} \frac{\partial(r w)}{\partial Z}, \\
& \tilde{u}_{\phi}=\frac{\partial \xi_{\phi}}{\partial T}+r U_{z} \frac{\partial}{\partial Z}\left(\frac{\xi_{\phi}}{r}\right), \\
& \frac{\partial \tilde{u}_{\perp}}{\partial T}+r U B_{z} \frac{\partial}{\partial Z}\left(\frac{\tilde{u}_{\perp}}{r B}\right)=\frac{r B B_{z}}{\mu_{0} \rho} \frac{\partial}{\partial Z}\left(\frac{B_{z}}{r^{2} B^{2}} \frac{\partial(r w)}{\partial Z}\right) \\
& \quad-\frac{r B^{3}}{\rho} \frac{\partial Q}{\partial \psi}+O\left(\epsilon^{2}\right), \\
& \frac{\partial \tilde{u}_{\phi}}{\partial T}+\frac{U_{z}}{r} \frac{\partial\left(r \tilde{u}_{\phi}\right)}{\partial Z}=\frac{B_{z}}{\mu_{0} \rho r} \frac{\partial}{\partial Z}\left[r^{2} B_{z} \frac{\partial}{\partial Z}\left(\frac{\xi_{\phi}}{r}\right)\right]-\frac{\mathrm{i} B^{2} Q}{\rho r} .
\end{aligned}
$$

Eliminating $\tilde{u}_{\perp}, \tilde{u}_{\phi}$, and $\xi_{\phi}$ and only keeping the leading terms with respect to $\epsilon$ we obtain the system of equations for $w$ and $Q$ 
in the leading order approximation:

$$
\begin{aligned}
Q= & \frac{1}{\mu_{0} B} \frac{\partial}{\partial Z}\left(r^{2} B \frac{\partial^{2} W}{\partial \psi \partial Z}\right) \\
& -\frac{\rho}{B^{2}}\left(r^{2} \frac{\partial}{\partial T}+U \frac{\partial}{\partial Z} r^{2}\right)\left(\frac{\partial^{2} W}{\partial T \partial \psi}+U \frac{\partial^{2} W}{\partial \psi \partial Z}\right), \\
\left(\frac{\partial}{\partial T}+\right. & \left.r^{2} U B^{2} \frac{\partial}{\partial Z} \frac{1}{r^{2} B^{2}}\right)\left(\frac{\partial W}{\partial T}+U \frac{\partial W}{\partial Z}\right) \\
& =\frac{r^{2} B^{3}}{\mu_{0} \rho} \frac{\partial}{\partial Z}\left(\frac{1}{r^{2} B} \frac{\partial W}{\partial Z}\right)-\frac{r^{2} B^{4}}{\rho} \frac{\partial Q}{\partial \psi},
\end{aligned}
$$

where $W=r w=r B \xi_{\perp}$. Using Eq. (43) and recalling that, outside of the transitional layer, we can consider $\rho, B$, and $U$ as quantities independent of $r$ and, consequently, on $\psi$, we reduce Eqs. (63) and (64) to

$Q=\frac{2 \psi}{h} \frac{\partial}{\partial \psi}\left[\frac{1}{\mu_{0}} \frac{\partial^{2} W}{\partial Z^{2}}-\frac{\rho}{h^{2}}\left(\frac{\partial}{\partial T}+h U \frac{\partial}{\partial Z} \frac{1}{h}\right)\left(\frac{\partial W}{\partial T}+U \frac{\partial W}{\partial Z}\right)\right]$,

$\left(\frac{\partial}{\partial T}+h U \frac{\partial}{\partial Z} \frac{1}{h}\right)\left(\frac{\partial W}{\partial T}+U \frac{\partial W}{\partial Z}\right)=\frac{h^{2}}{\mu_{0} \rho} \frac{\partial^{2} W}{\partial Z^{2}}-\frac{2 \psi h^{3}}{\rho} \frac{\partial Q}{\partial \psi}$

We differentiate Eq. (66) with respect to $\psi$ and then eliminate $W$ from the obtained equation and Eq. (65). This yields

$4 \psi \frac{\partial}{\partial \psi} \psi \frac{\partial Q}{\partial \psi}-Q=0$

The solution to this equation must be regular at $\psi=0$ and decay as $\psi \rightarrow \infty$. Hence, we obtain

$$
Q=\left\{\begin{array}{lc}
Q_{i}(T, Z) \psi^{1 / 2}, & 0 \leq \psi \leq \psi_{i}, \\
Q_{e}(T, Z) \psi^{-1 / 2}, & \psi \geq \psi_{e}
\end{array}\right.
$$

where $Q_{i}(T, Z)$ and $Q_{e}(T, Z)$ are arbitrary functions. Recall that $\psi=\psi_{i}$ and $\psi=\psi_{e}$, where $\psi_{i}$ and $\psi_{e}$ are constants, are the equations of the boundaries of the transitional layer. It follows from Eq. (43) that

$\psi_{i}=\frac{C}{2}\left(1-\frac{l}{2}\right)^{2}, \quad \psi_{e}=\frac{C}{2}\left(1+\frac{l}{2}\right)^{2}$.

Equations (66) and (68) imply that $W=\psi^{1 / 2} \widetilde{W}(T, Z)$ inside the tube core region. Then, recalling the definition of $W$ and using Eq. (43) we conclude that $\xi_{\perp}$ is independent of $r$ inside the tube core region, which implies that, in the thin tube approximation, the tube oscillates as a solid.

Substituting Eq. (68) in Eq. (66) we arrive at 10pt

$$
\begin{aligned}
& \left(\frac{\partial}{\partial T}+h U_{i} \frac{\partial}{\partial Z} \frac{1}{h}\right)\left(\frac{\partial W_{i}}{\partial T}+U_{i} \frac{\partial W_{i}}{\partial Z}\right)-\frac{h^{2}}{\mu_{0} \rho_{i}} \frac{\partial^{2} W_{i}}{\partial Z^{2}}=-\frac{\psi_{i}^{1 / 2} h^{3} Q_{i}}{\rho_{i}} \\
& \left(\frac{\partial}{\partial T}+h U_{e} \frac{\partial}{\partial Z} \frac{1}{h}\right)\left(\frac{\partial W_{e}}{\partial T}+U_{e} \frac{\partial W_{e}}{\partial Z}\right)-\frac{h^{2}}{\mu_{0} \rho_{e}} \frac{\partial^{2} W_{e}}{\partial Z^{2}}=\frac{h^{3} Q_{e}}{\rho_{e} \psi_{e}^{1 / 2}}
\end{aligned}
$$

where $W_{i}$ and $W_{e}$ are calculated at $\psi=\psi_{i}$ and $\psi=\psi_{e}$, respectively. We introduce the new variable

$\eta=\left.\frac{1}{R(z)} \xi_{\perp}\right|_{\psi=\psi_{i}}$.

We also introduce the jumps of this new variable and the magnetic pressure perturbation across the transitional layer,

$\delta \eta=\left.\eta\right|_{\psi=\psi_{e}}-\left.\eta\right|_{\psi=\psi_{i}}, \quad \delta P=\left.P\right|_{\psi=\psi_{e}}-\left.P\right|_{\psi=\psi_{i}}$.
We have the estimates $\delta \eta=O(l), \delta P=O(l)$. Then, with the aid of Eq. (68), we obtain

$$
\begin{gathered}
W_{i}=C(1-l / 2) \eta, \quad W_{e}=C[(1+l / 2) \eta+\delta \eta] \\
\psi_{i}^{1 / 2} h^{3} Q_{i}=\left.\frac{\epsilon^{-2} C}{R^{2}} P\right|_{\psi=\psi_{i}}, \quad \psi_{e}^{-1 / 2} h^{3} Q_{e}=\left.\frac{\epsilon^{-2} C}{R^{2}} P\right|_{\psi=\psi_{e}}
\end{gathered}
$$

Now we multiply Eq. (70) by $\rho_{i}$, Eq. (71) by $\rho_{e}$, and add the results; use Eqs. (46), (73), and (74); and return to the original non-scaled independent variables. As a result, we arrive at

$$
\begin{aligned}
\rho_{i}\left(\frac{\partial}{\partial t}+\frac{U_{i}}{R^{2}} \frac{\partial}{\partial z} R^{2}\right)\left(\frac{\partial \eta}{\partial t}+U_{i} \frac{\partial \eta}{\partial z}\right) \\
+\rho_{e}\left(\frac{\partial}{\partial t}+\frac{U_{e}}{R^{2}} \frac{\partial}{\partial z} R^{2}\right)\left(\frac{\partial \eta}{\partial t}+U_{e} \frac{\partial \eta}{\partial z}\right)-\frac{2 B^{2}}{\mu_{0}} \frac{\partial^{2} \eta}{\partial z^{2}}=\mathcal{L} \\
\mathcal{L}=\frac{\delta P}{R^{2}}+\frac{B^{2}}{\mu_{0}} \frac{\partial^{2}(l \eta+\delta \eta)}{\partial z^{2}} \\
\quad-\rho_{e}\left(\frac{\partial}{\partial t}+\frac{U_{e}}{R^{2}} \frac{\partial}{\partial z} R^{2}\right)\left(\frac{\partial}{\partial t}+U_{e} \frac{\partial}{\partial z}\right)(l \eta+\delta \eta)
\end{aligned}
$$

We note that we only kept terms of the order of $l$ when deriving Eq. (76), while we neglected terms of higher orders with respect to $l$. When there is no transitional layer $(l=0)$ we have $\mathcal{L}=0$, and Eq. (75) governs the kink oscillations of a thin expanding magnetic tube with a background field-aligned flow. However, in the presence of the transitional layer the system of Eqs. (75) and (76) is not closed. To close it one needs to express $\delta \eta$ and $\delta P$ in terms of $\eta$. We do not do this here. These expressions are obtained when we use Eqs. (75) and (76) to study the damping of kink oscillations of expanding magnetic flux tubes due to resonant absorption.

When there is no flow $(U=0)$ and no transitional region $(l=0)$, Eq. (75) reduces to the equation describing the kink oscillations of a thin expanding magnetic tube derived by Ruderman et al. (2008). On the other hand, when there is no expansion ( $R=$ const.), Eqs. (75) and (76) reduce to Eqs. (23) and (24) in Ruderman (2011a) describing resonantly damped kink oscillations of a thin magnetic tube with non-stationary density and flow. We note that the expression for $\mathcal{L}$ given by Eq. (76) with $R=$ const. is slightly different from that given by Equations (24) in Ruderman (2011a). However, it is straightforward to show using Eq. (75) that the difference between $R \mathcal{L}$ with $\mathcal{L}$ given by Eq. (76) and $\mathcal{L}$ given by Eqs. (24) in Ruderman (2011a) is of the order of $l^{2}$.

It is expedient to compare the results of this section with that obtained by Andries \& Cally (2011). They considered waves in static magnetic tubes with sharp boundaries. They assumed that the characteristic scale of the tube cross-section variation along the tube is much larger than the characteristic transverse scale of the tube; however, they did not assume that the perturbation wavelength is much larger than the characteristic transverse scale. In particular, they managed to obtain a single equation describing kink oscillations. However, this equation is quite complicated because it contains functions of differential operators. It is quite difficult to work with them. We see that the thin tube approximation enormously simplifies the study of kink oscillations. Even when the tube is non-stationary and there is background flow, kink oscillations are described by a relatively simple differential Eq. (75). 


\section{Eigenvalue problem in the presence of stationary flow}

In what follows we consider kink oscillations of magnetic tubes with the sharp boundary and take $l=0$. We assume that there is no flow outside the tube, $U_{e}=0$. We also assume that both the density and background flow velocity are independent of time. Then Eq. (52) reduces to

$\rho_{i} U R^{2}=$ const.,

where we have dropped the subscript " $i$ " at the internal flow speed $U$. Using Eq. (45) we rewrite Eq. (46) in the approximate form

$B R^{2}=$ const.

Since we have taken $l=0$ it follows that $\mathcal{L}=0$. Below we study standing waves and impose the boundary conditions

$\eta=0 \quad$ at $\quad z= \pm L / 2$,

where $L$ is the tube length. We look for eigenfrequencies of kink oscillations, so we take $\eta \propto \mathrm{e}^{-\mathrm{i} \omega t}$. Then Eq. (75) with $\mathcal{L}=0$ reduces to

$$
\begin{aligned}
\left(\frac{2 B^{2}}{\mu_{0}}-\rho_{i} U^{2}\right) \frac{\mathrm{d}^{2} \eta}{\mathrm{d} z^{2}} & +\left(2 \mathrm{i} \omega \rho_{i} U-\frac{\rho_{i} U}{R^{2}} \frac{\mathrm{d}\left(U R^{2}\right)}{\mathrm{d} z}\right) \frac{\mathrm{d} \eta}{\mathrm{d} z} \\
& +\left(\frac{2 \mathrm{i} \omega \rho_{i} U}{R} \frac{\mathrm{d} R}{\mathrm{~d} z}+\omega^{2}\left(\rho_{i}+\rho_{e}\right)\right) \eta=0 .
\end{aligned}
$$

This equation, together with the boundary conditions Eq. (79), constitutes an eigenvalue problem. The aim of this section is to study general properties of this problem. Below we assume that $\mu_{0} \rho_{i} U^{2}<2 B^{2}$, that is $U^{2}<2 V_{A i}^{2} \equiv 2 B^{2} / \mu_{0} \rho_{i}$, and this condition is satisfied for all $z \in[-L / 2, L / 2]$. Then we make the variable substitution

$\eta=q \exp (-\mathrm{i} \omega \sigma(z)), \quad \sigma=\int_{-L / 2}^{z} \frac{\mu_{0} \rho_{i} U \mathrm{~d} z^{\prime}}{2 B^{2}-\mu_{0} \rho_{i} U^{2}}$.

Now, multiplying the obtained equation by $R^{4}$, we reduce Eq. (80) to

$\frac{\mathrm{d}}{\mathrm{d} z}\left[R^{4}\left(\frac{2 B^{2}}{\mu_{0}}-\rho_{i} U^{2}\right) \frac{\mathrm{d} q}{\mathrm{~d} z}\right]+\omega^{2} W(z) q=0$,

where

$W(z)=R^{4} \frac{2\left(\rho_{i}+\rho_{e}\right) B^{2}-\mu_{0} \rho_{i} \rho_{e} U^{2}}{2 B^{2}-\mu_{0} \rho_{i} U^{2}}$.

Equation (82) with the boundary conditions Eq. (79) constitutes the classical Sturm-Liouville problem with the eigenvalue $\omega^{2}$. It follows from the general theory of the Sturm-Liouville problem (e.g. Coddington \& Levinson 1955) that the eigenvalues are real and constitute a monotonically increasing unbounded sequence. The first eigenvalue is the square of the fundamental frequency, and the corresponding eigenfunction has no nodes in $(-L / 2, L / 2)$. All other eigenvalues are the squares of frequencies of overtones. The eigenfunction corresponding to the $n$th overtone has $n-1$ nodes in $(-L / 2, L / 2)$.

It is obvious that we can always take $q$ to be real. Multiplying Eq. (82) by $q$, integrating the obtained equation, and using Eq. (79) we obtain

$\omega^{2} \int_{-L / 2}^{L / 2} W(z) q^{2} \mathrm{~d} z=\int_{-L / 2}^{L / 2} R^{4}\left(\frac{2 B^{2}}{\mu_{0}}-\rho_{i} U^{2}\right)\left(\frac{\mathrm{d} q}{\mathrm{~d} z}\right)^{2} \mathrm{~d} z$.
Since $W(z)>0$, it follows from this equation that $\omega^{2}>0$, i.e. that all the eigenfrequencies are real. This implies that the inequality $U^{2}<2 V_{A i}^{2}$ is a sufficient condition for the stability of the tube with respect to long kink perturbations. We emphasise that this condition is sufficient but not necessary. On the other hand, this analysis does not prove that the tube is stable if the condition $U^{2}<2 V_{A i}^{2}$ is satisfied. While it guaranties that the tube is stable with respect to long kink perturbations, it can be unstable with respect to other types of perturbations.

\section{Kink oscillations of coronal loops with slowly varying density}

The analysis in this section is motivated by the observations of kink oscillations of cooling coronal loops (e.g. Aschwanden \& Terradas 2008; Aschwanden \& Schrijver 2011). Below we consider kink oscillations of a magnetic tube with slowly varying density. We assume that the boundary condition Eq. (79) is satisfied. We also assume that the characteristic time of the density variation, $t_{\mathrm{ch}}$, is much greater than the characteristic time of oscillations. We note that this is not a very restrictive assumption. It is usually believed that the characteristic time of oscillations is the oscillation period. However, this is not true. The characteristic time of oscillations is $1 / \omega$, where $\omega$ is the oscillation frequency. This time is about 6 times smaller than the oscillation period. Hence, the condition that $t_{\mathrm{ch}}$ should be much larger than the characteristic time of oscillations is satisfied even when $t_{\mathrm{ch}}$ is of the order of the oscillation period. We also assume that the loop has a sharp boundary, which is $l=0$ and, consequently, $\mathcal{L}=0$.

\subsection{Derivation of adiabatic invariant}

We denote the ratio of $t_{\mathrm{ch}}$ to the characteristic time of oscillations as $v^{-1}$, where $v \ll 1$. Hence, the characteristic time of oscillations is $v t_{\mathrm{ch}}$. On the other hand, it is also of the order of the loop length divided by the characteristic kink speed, which is equal to $L /\left(B / \sqrt{\mu_{0} \rho_{\mathrm{ch}}}\right)$, where $\rho_{\mathrm{ch}}$ is the characteristic density. As a result, we have

$B \sim v^{-1} \sqrt{\mu_{0} \rho_{\mathrm{ch}}} \frac{L}{t_{\mathrm{ch}}}$.

This estimate inspired us to introduce the scaled magnetic field $\widetilde{B}=v B$. Then we rewrite Eq. (75) with $\mathcal{L}=0$ as

$$
\begin{aligned}
\rho_{i}\left(\frac{\partial}{\partial t}+\right. & \left.\frac{U_{i}}{R^{2}} \frac{\partial}{\partial z} R^{2}\right)\left(\frac{\partial \eta}{\partial t}+U_{i} \frac{\partial \eta}{\partial z}\right) \\
& +\rho_{e}\left(\frac{\partial}{\partial t}+\frac{U_{e}}{R^{2}} \frac{\partial}{\partial z} R^{2}\right)\left(\frac{\partial \eta}{\partial t}+U_{e} \frac{\partial \eta}{\partial z}\right)-\frac{2 v^{-2} \widetilde{B}^{2}}{\mu_{0}} \frac{\partial^{2} \eta}{\partial z^{2}}=0 .
\end{aligned}
$$

Now we use the Wentzel-Kramer-Brillouin (WKB) method (e.g. Bender \& Orszag 1978) and look for the solution to this equation in the form

$\eta=S(t, z) \exp \left[v^{-1} \Theta(t)\right]$.

Then we expand $S$ in the series

$S=S_{0}+v S_{1}+\ldots$

Substituting Eq. (86) in Eq. (85), using Eq. (87), collecting terms of the order of $v^{-2}$, and taking into account the boundary condition Eq. (79) we obtain the Sturm-Liouville problem

$\frac{\partial^{2} S_{0}}{\partial z^{2}}+\frac{\Omega^{2}}{\widetilde{C}_{k}^{2}} S_{0}=0, \quad S_{0}=0$ at $z= \pm L / 2$, 
where

$\Omega=\frac{\mathrm{d} \Theta}{\mathrm{d} t}, \quad \widetilde{C}_{k}^{2}=\frac{2 \widetilde{B}^{2}}{\mu_{0}\left(\rho_{i}+\rho_{e}\right)}$.

This order approximation is called the approximation of geometrical optics (e.g. Bender \& Orszag 1978). The SturmLiouville problem Eq. (88) coincides with a problem obtained by Dymova \& Ruderman (2005) to describe the kink oscillations of magnetic tubes whose density varies along the tube, and by Ruderman et al. (2008) to describe the kink oscillations of magnetic tubes whose density and cross-section radius vary along the tube. Below we assume that $\Omega^{2}$ is an eigenvalue and $S_{0}$ the corresponding eigenfunction. In accordance with the general theory (e.g. Coddington \& Levinson 1955), $\Omega^{2}$ is real. It is easy to prove that $\Omega^{2}>0$. Obviously we can always assume that $S_{0}$ is a real function.

Now we collect the terms of the order of $v^{-1}$ to obtain

$\frac{\partial^{2} S_{1}}{\partial z^{2}}+\frac{\Omega^{2}}{\widetilde{C}_{k}^{2}} S_{1}=\frac{2 \mathrm{i} \Omega}{\widetilde{C}_{k}^{2}}\left(\frac{\partial S_{0}}{\partial t}+\frac{S_{0}}{2 \Omega} \frac{\partial \Omega}{\partial t}+\frac{\rho_{i} U_{i}+\rho_{e} U_{e}}{R\left(\rho_{i}+\rho_{e}\right)} \frac{\partial\left(R S_{0}\right)}{\partial z}\right)$.

This is the approximation of the physical optics. $S_{1}$ must satisfy the boundary conditions

$S_{1}=0 \quad$ at $\quad z= \pm L / 2$.

The homogeneous counterpart of the boundary value problem constituted by Eq. (90) and the boundary conditions Eq. (91) has a non-trivial solution $S_{1}=S_{0}$. This implies that the boundary value problem determining $S_{1}$ has a solution only if the righthand side of Eq. (90) satisfies the compatibility condition, which is the condition that it should be orthogonal to $S_{0}$. To obtain this condition we multiply Eq. (90) by $S_{0}$ and then integrate with respect to $z$ from $-L / 2$ to $L / 2$. After some algebra we obtain

$\int_{-L / 2}^{L / 2} \frac{1}{\widetilde{C}_{k}^{2}} \frac{\partial\left(\Omega S_{0}^{2}\right)}{\partial t} \mathrm{~d} z=-\frac{\mu_{0} \Omega}{2} \int_{-L / 2}^{L / 2} \frac{\rho_{i} U_{i}+\rho_{e} U_{e}}{R^{2} \widetilde{B}^{2}} \frac{\partial\left(S_{0}^{2} R^{2}\right)}{\partial z} \mathrm{~d} z$.

Using the integration by parts, Eq. (52), and the relation $R^{2} \widetilde{B}=$ const. yields

$$
\int_{-L / 2}^{L / 2} \frac{\rho_{i} U_{i}+\rho_{e} U_{e}}{R^{2} \widetilde{B}^{2}} \frac{\partial\left(S_{0}^{2} R^{2}\right)}{\partial z} \mathrm{~d} z=\int_{-L / 2}^{L / 2} \frac{S_{0}^{2}}{\widetilde{B}^{2}} \frac{\partial\left(\rho_{i}+\rho_{e}\right)}{\partial t} \mathrm{~d} z .
$$

Substituting Eq. (93) in Eq. (92) and using Eq. (89) we eventually obtain

$\omega \int_{-L / 2}^{L / 2} \frac{S_{0}^{2}}{C_{k}^{2}} \mathrm{~d} z=$ const.

where

$\omega=v^{-1} \Omega, \quad C_{k}^{2}=v^{-2} \widetilde{C}_{k}^{2}=\frac{2 B^{2}}{\mu_{0}\left(\rho_{i}+\rho_{e}\right)}$.

The left-hand side of Eq. (94) is the adiabatic invariant. Equation (94) states that this invariant is conserved. It is worth noting that the adiabatic invariant is the same as that obtained by Ruderman (2011b) for oscillations of magnetic tubes with the constant cross-section radius. Hence, the tube expansion only affects the time evolution of kink oscillations of magnetic tubes with the varying density through $C_{k}$ and $S_{0}$ that both depend on $R(z)$.

\subsection{Effect of cooling on the kink oscillations of coronal magnetic loops}

In this subsection we consider the kink oscillations of cooling coronal loops. The main cause of the loop cooling is radiation. Its intensity is proportional to the plasma density squared. This implies that the energy deposition that is sufficient to cover the energy losses in the rarefied plasma outside the loop may be too small to cover the energy losses inside the loop. Hence, it seems to be a viable assumption that while the plasma inside the loop is cooling, the temperature of the plasma outside the loop remains constant. Following Aschwanden \& Terradas (2008) and Morton \& Erdélyi (2010), we approximate the temperature evolution inside the loop by an exponentially decaying function,

$T(t)=T_{0} \exp \left(-t / t_{\text {cool }}\right)$,

where we have assumed that the cooling starts at the initial time $t=0$. We also assume that the plasma temperature inside and outside the loop was the same at $t=0$, implying that the temperature of the external plasma remains equal to $T_{0}$.

Below we consider loops with a half-circle shape. We neglect the effect of the loop curvature on kink oscillations. Hence, the loop shape only determines the density variation along the loop. In the external plasma it is given by the barometric formula

$\rho_{e}(z)=\frac{\rho_{f}}{\zeta} \exp \left(-\frac{L}{\pi H_{0}} \cos \frac{\pi z}{L}\right)$

where $\rho_{f}$ is the plasma density at the footpoints inside the loop (assumed to be constant), and $\zeta$ the ratio of the plasma densities inside and outside the loop at $t=0$,

$H_{0}=\frac{k_{\mathrm{B}} T_{0}}{m g}$

$k_{\mathrm{B}}$ is the Boltzmann constant, $g$ the gravity acceleration, and $m$ the mean mass per particle equal to one half of the proton mass for a proton-electron plasma. The cooling causes the plasma flow inside the loop. This flow affects the plasma density meaning that it is not described by the barometric formula. However, Ruderman (2011b) showed that for typical coronal conditions and observed cooling times the effect of this flow on the plasma density is fairly week. Hence, the barometric formula provides a sufficiently good approximation for the plasma density inside the loop. It reads

$\rho_{i}(z)=\rho_{f} \exp \left(-\frac{L}{\pi H(t)} \cos \frac{\pi z}{L}\right)$,

where $H(t)$ is defined by Eq. (98) with $T(t)$ substituted for $T_{0}$.

To describe the cross-section radius variation along the loop we use the same expression as in Ruderman et al. (2008),

$R(z)=R_{f} \lambda \sqrt{\frac{\cosh \left(L / 2 l_{\mathrm{c}}\right)-1}{\cosh \left(L / 2 l_{\mathrm{c}}\right)-\lambda^{2}+\left(\lambda^{2}-1\right) \cosh \left(z / l_{\mathrm{c}}\right)}}$,

where $R_{f}$ is the cross-section radius at the footpoints, $l_{\mathrm{c}}$ an arbitrary positive constant with the dimension of length, and $\lambda=R(0) / R_{f}$ the expansion factor. It is shown in Ruderman et al. (2008) that in order to have the $z$-component of the magnetic field positive everywhere in the region $|z| \leq L / 2$, the expansion factor must satisfy the inequality $\lambda<\lambda_{\mathrm{m}}$, where

$\lambda_{\mathrm{m}}^{2} \approx \frac{1.4 \cosh \left(L / 2 l_{\mathrm{c}}\right)}{1+0.4 \cosh \left(L / 2 l_{\mathrm{c}}\right)}$ 
The parameter $\lambda_{\mathrm{m}}$ is a monotonically increasing function of $L / l_{\mathrm{c}}$, $\lambda_{\mathrm{m}} \rightarrow 1$ as $L / l_{\mathrm{c}} \rightarrow 0$, and $\lambda_{\mathrm{m}} \rightarrow 1.87$ as $L / l_{\mathrm{c}} \rightarrow \infty$. As we have already pointed out, the coronal loop expansion factor typically does not exceed 1.5 . Hence, by properly choosing the ratio $L / l_{\mathrm{p}}$ we can cover the whole range of the expansion factor variation.

We introduce the dimensionless variables and parameters

$Z=\frac{2 z}{L}, \tau=\frac{t}{t_{\mathrm{cool}}}, \varpi=\frac{\omega L}{C_{f}}, \kappa=\frac{L}{\pi H_{0}}, \Lambda=\frac{R}{R_{f}}, S_{0}^{\prime}=\frac{S_{0}}{R_{f}}$,

where the kink speed at the footpoints is defined by

$C_{f}^{2}=\frac{2 \zeta B_{f}^{2}}{\mu_{0} \rho_{f}(\zeta+1)}$.

Then Eq. (88) reduces to

$$
\begin{gathered}
\frac{\partial^{2} S_{0}}{\partial Z^{2}}+\frac{\varpi^{2} \Lambda^{4}(Z) S_{0}}{4(\zeta+1)}\left[\zeta \exp \left(-\kappa e^{\tau} \cos (\pi Z / 2)\right)\right. \\
+\exp (-\kappa \cos (\pi Z / 2))]=0 .
\end{gathered}
$$

When deriving this equation we dropped the prime at $S_{0}$ and used the relation $B \Lambda^{2}=B_{f}$. The function $\Lambda(Z)$ is defined by Eq. (100). The eigenfunction corresponding to the fundamental mode is even, while the eigenfunction corresponding to the first overtone is odd. This enables us to solve Eq. (104) not on the interval $[-1,1]$, but on the interval $[0,1]$ The solution corresponding to the fundamental mode must satisfy the boundary conditions

$\frac{\partial S_{0}}{\partial Z}=0$ at $Z=0, \quad S_{0}=0$ at $Z=1$,

and the solution corresponding to the first overtone must satisfy the boundary conditions

$S_{0}=0$ at $Z=0,1$.

Since the eigenfunction $S_{0}$ is accurately determined up to the multiplication by an arbitrary function of $\tau$, we can always fix its value at one particular point. Let $X(\tau, Z)$ be an eigenfunction corresponding to the fundamental mode that satisfies the condition $X(\tau, 0)=1$. Then the general solution to the eigenvalue problem corresponding to the fundamental mode is $S_{0}(\tau, Z)=$ $A(\tau) X(\tau, Z)$, where $A(\tau)$ is the oscillation amplitude at $Z=0$. Then Eq. (94) reduces to

$$
\begin{gathered}
\varpi A^{2} \int_{-1}^{1} X^{2} \Lambda^{4}\left[\zeta \exp \left(-\kappa \mathrm{e}^{\tau} \cos (\pi Z / 2)\right)\right. \\
+\exp (-\kappa \cos (\pi Z / 2))] \mathrm{d} Z=\text { const. }
\end{gathered}
$$

Equations (104) and (107) have been solved numerically for $\zeta=3$ and $l_{\mathrm{c}}=L / 6$. In this case $\lambda_{\mathrm{m}} \approx 1.67$. In Figs. $1-3$ the dependences of the dimensionless fundamental mode frequency, the ratio of frequencies of the first overtone and fundamental mode, and the oscillation amplitude at $Z=0$ on $\tau$ are shown for various values of $\kappa$ and $\lambda$.

We see in Fig. 1 that the oscillation frequency grows for all values of the expansion factor and for all values of the loop height. The same result was obtained by Morton \& Erdélyi (2009, 2010) and Ruderman (2011b) for non-expanding magnetic loops. This result has a simple physical explanation. When the loop is cooling the plasma flow with the velocity directed to the loop footpoints appears. The plasma is evacuated from the
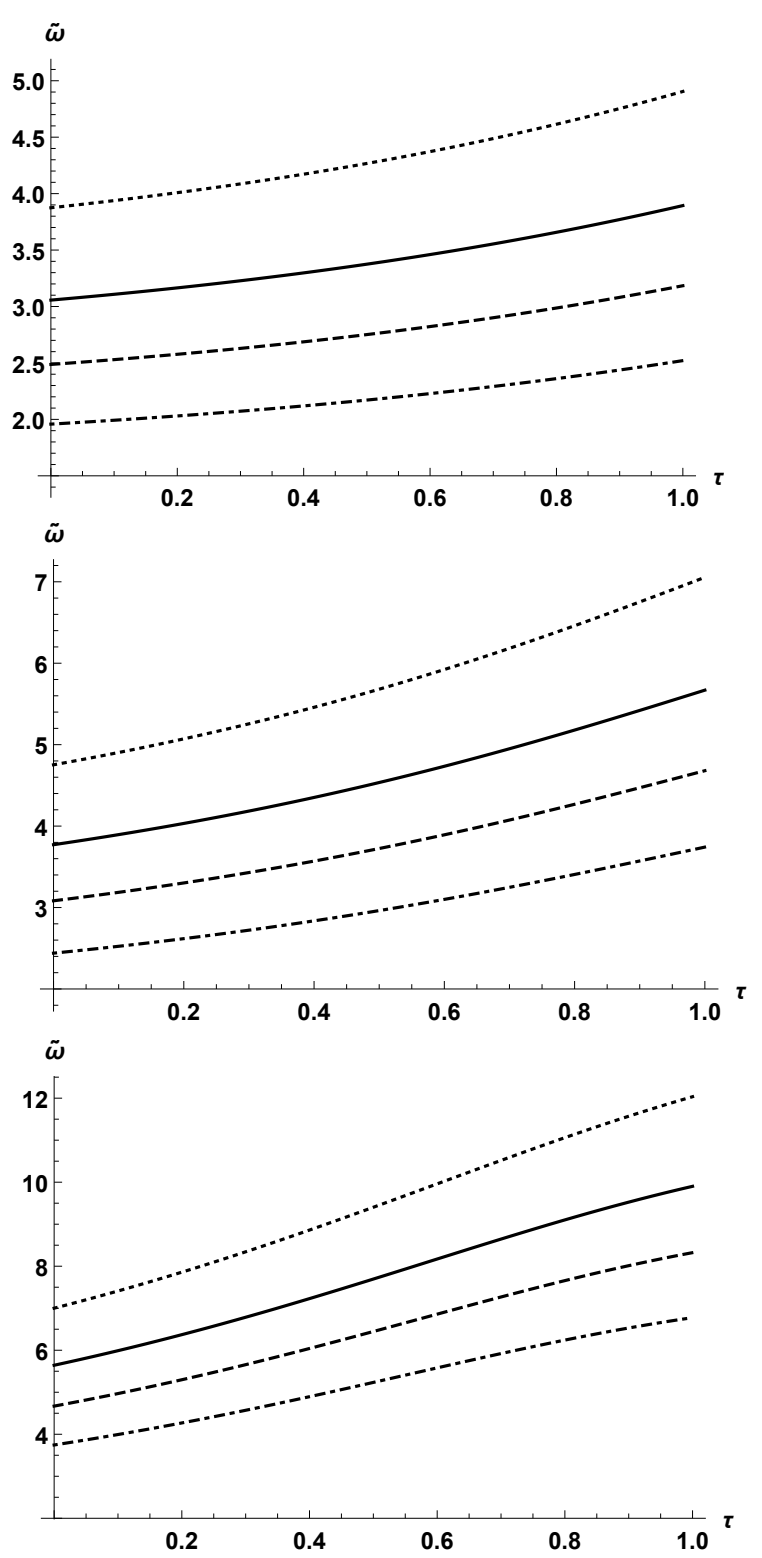

Fig. 1. Dependence of the dimensionless fundamental mode frequency on $\tau$. The upper, middle, and lower panels correspond to $\kappa=0.5, \kappa=1$, and $\kappa=2$. The dotted, solid, dashed, and dash-dotted lines correspond to $\lambda=1,1.15,1.3$, and 1.5 , respectively.

loop meaning that the loop mass decreases. At the same time the restoring force created by the magnetic tension remains the same. As a result the oscillation frequency increases.

Figure 2 shows that the ratio of frequencies of the first overtone and fundamental harmonic decreases with time. Again the same result was obtained by Morton \& Erdélyi (2009, 2010) and Ruderman (2011b) for non-expanding magnetic loops. And again there is a simple physical explanation for this result. Owing to cooling, the plasma density at the loop decreases everywhere except for the loop footpoints. In particular, it decreases at the loop apex. As a result the kink speed at the loop apex increases, while it remains the same at the loop footpoints. Ruderman et al. (2016) showed that the frequency ratio is a monotonically decreasing function of the ratio of the kink speed at the loop apex and footpoints.

The most interesting result of this section is shown in Fig. 3. We see that cooling enhances the oscillation amplitude. Previously the same result was obtained by Ruderman (2011b) for 


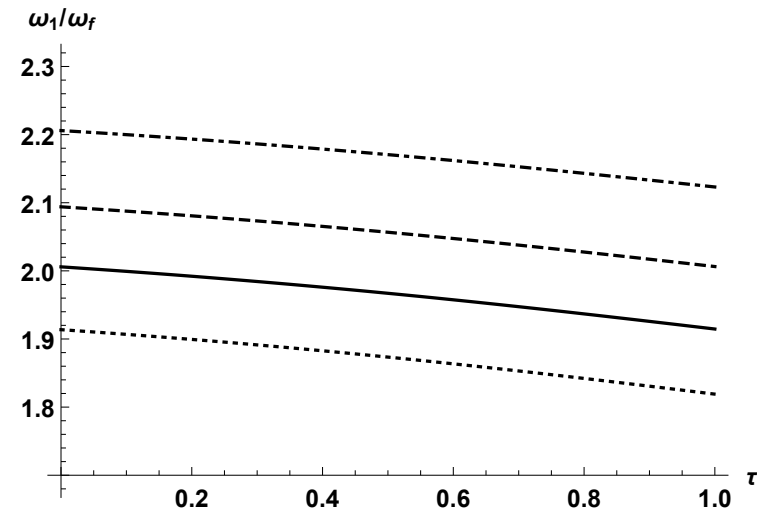

$\omega_{1} / \omega_{f}$
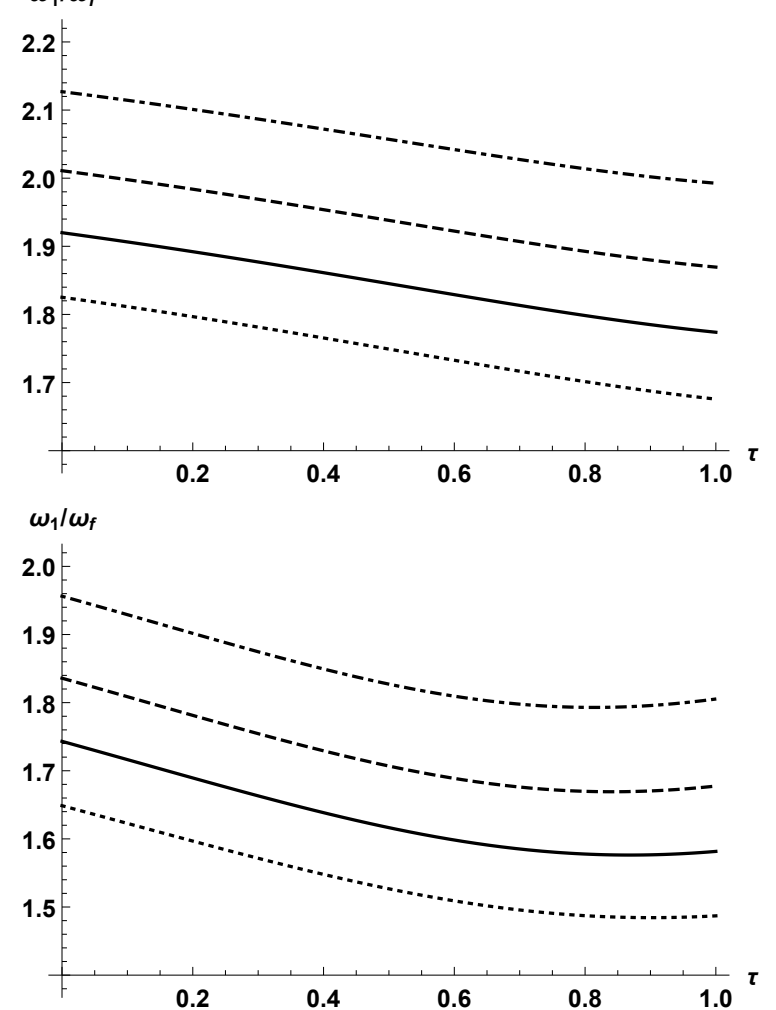

Fig. 2. Dependence of the ratio of frequencies of the first overtone and fundamental mode on $\tau$. The upper, middle, and lower panels correspond to $\kappa=0.5, \kappa=1$, and $\kappa=2$. The dotted, solid, dashed, and dash-dotted lines correspond to $\lambda=1,1.15,1.3$, and 1.5 , respectively.

non-expanding loops. We also see that the loop expansion makes the oscillation amplification stronger. This effect becomes more pronounced when the loop height increases. However, even for loops whose height is equal to two times the initial atmospheric scaleheight, the effect is fairly weak.

\section{Summary and conclusions}

In this article, we studied kink waves and oscillations of thin expanding magnetic tubes in the presence of flow. Both the plasma density and flow velocity were allowed to vary along the tube and depend on time. These physical quantities can also vary in the radial direction. However, it is assumed that the characteristic scale of their variation in the radial direction in the core region inside the tube and in the region outside the tube is the same as that along the loop. They can only vary on short spatial scales inside a thin transitional layer at the tube boundary.
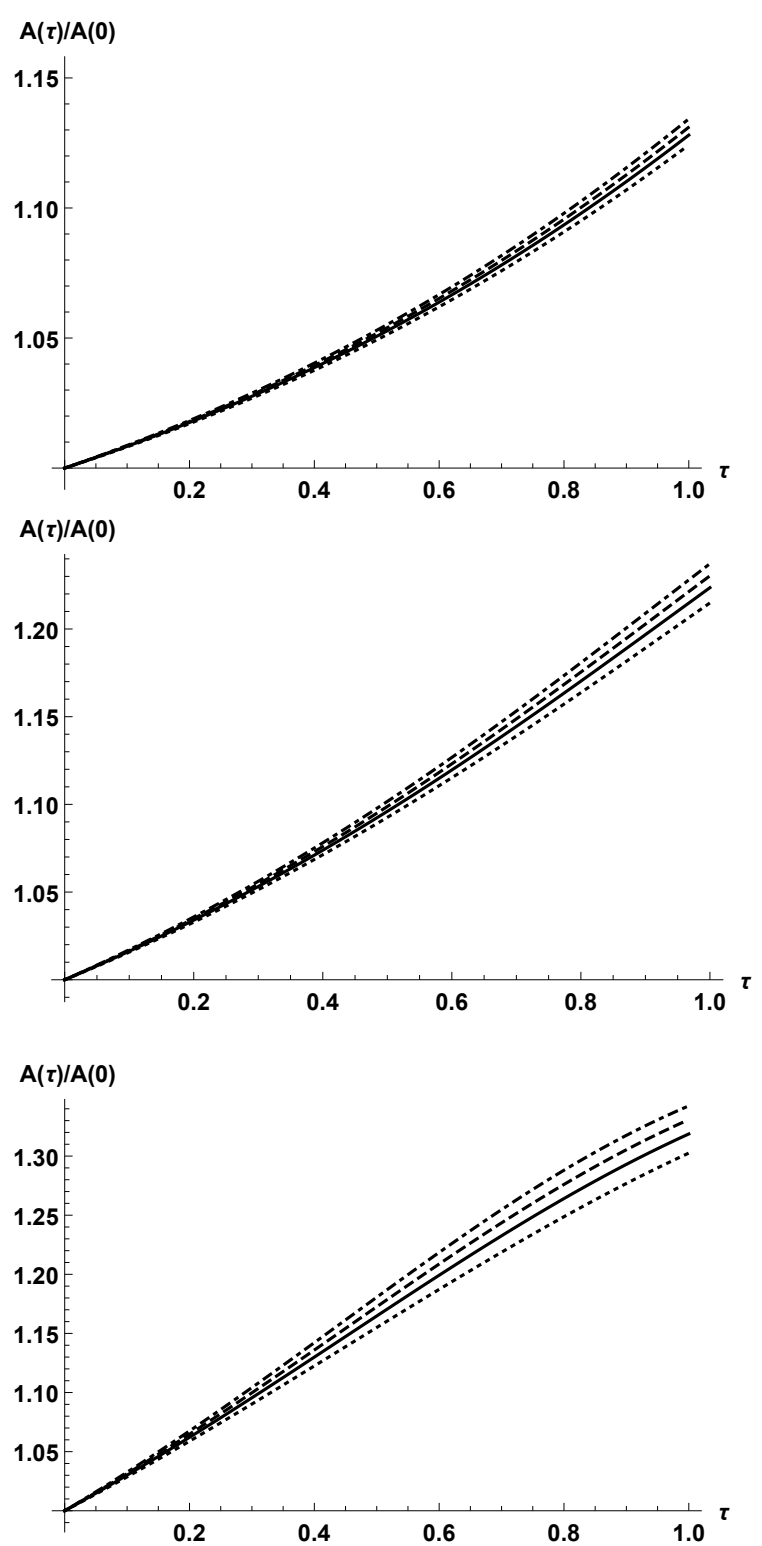

Fig. 3. Dependence of the dimensionless amplitude of the fundamental mode at $Z=0$ on $\tau$. The upper, middle, and lower panels correspond to $\kappa=0.5, \kappa=1$, and $\kappa=2$. The dotted, solid, dashed, and dash-dotted lines correspond to $\lambda=1,1.15,1.3$, and 1.5 , respectively.

Using the multiscale expansion we derived the system of two equations (see Eqs. (75) and (76)) governing the kink waves and oscillations. This is the main result of the article. We emphasise that this system describes both standing and propagating waves. When there is no transitional layer the oscillations are only governed by the first equation. On the other hand, in the presence of the transitional layer the system of two equations is not closed because it contains the jumps of the plasma displacement and magnetic pressure across the transitional layer. To close this system these jumps must be expressed in terms of the tube displacement.

We used the derived equations to study the effect of the plasma density variation with time. We assumed that the characteristic time of the density variation is much greater than the characteristic time of oscillations. Then we used the WKB method to derive the expression for the adiabatic invariant, which is the quantity that is conserved while the plasma density changes. The general theory was applied to kink oscillations 
of cooling coronal loops. We considered expanding loops with a half-circle shape and assumed that the plasma temperature inside the loop decays exponentially. Then we numerically calculated the time dependence of the fundamental mode frequency, the ratio of frequencies of the first overtone and fundamental mode, and the oscillation amplitude.

The numerical results can be summarised as follows. Cooling causes an increase in the oscillation frequency and a decrease in the ratio of frequencies of the first overtone and fundamental mode. This is the generalisation of the results previously obtained by Morton \& Erdélyi (2009, 2010) and Ruderman (2011b) for non-expanding loops. Cooling also causes the enhancement of the oscillation amplitude. Previously, this result was obtained by Ruderman (2011b) for non-expanding loops. The amplitude enhancement due to cooling becomes stronger when the loop expansion increases. This effect is more pronounced for higher loops. However, it is very moderate even for loops where the height is equal to the two times the initial atmospheric scale height.

Acknowledgements. M.S.R. and R.E. acknowledge the support by the STFC grant. R.E. acknowledges the support received from the Chinese Academy of Sciences President's International Fellowship Initiative, Grant No. 2016VMA045. R.E. also acknowledges the excellent hospitality of the National Astronomical Observatory of China (NAOC), Chinese Academy of Sciences (CAS), where a part of this work was carried out.

\section{References}

Al-Ghafri, K. S., \& Erdélyi, R. 2013, Sol. Phys., 283, 413

Al-Ghafri, K. S., Ruderman, M. S., Williamson, A., \& Erdélyi, R. 2014, ApJ, 786, A36

Andries, J., \& Cally, P. S. 2011, ApJ, 743, 164

Andries, J., Arregui, I., \& Goossens, M. 2005a, ApJ, 624, L57

Andries, J., Goossens, M., Hollweg, J. V., Arregui, I., \& Van Doorsselaere, T. 2005b, A\&A, 430, 1109
Andries, J., Van Doorsselaere, T., Roberts, B., et al. 2009, Space Sci. Rev., 149,

Aschwanden, M. J., \& Schrijver, C. J. 2011, ApJ, 736, A102

Aschwanden, M. J., \& Terradas, J. 2008, ApJ, 686, L127

Aschwanden, M. J., Fletcher, L., Schrijver, C. J., \& Alexander, D. 1999, ApJ, 520,880

Bender, C. M., \& Orszag, S. A. 1978, Advanced Mathematical Methods for Scientists and Engineers (New York: McGraw-Hill)

Brekke, P., Kjeldseth-Moe, O., \& Harrison, R. A. 1997, Sol. Phys., 175, 511

Chae, J., Ahn, K., Lim, E. K., Choe, G. S., \& Sakurai, T. 2008, ApJ, 689, L73

Coddington, E. A., \& Levinson, N. 1955, Theory of Ordinary Differential Equations (New York: McGraw-Hill)

Dymova, M. V., \& Ruderman, M. S. 2005, Sol. Phys., 229, 79

Edwin, P. M., \& Roberts, B. 1983, Sol. Phys., 88, 179

Erdélyi, R., Hague, A., \& Nelson, C. J. 2014, Sol. Phys., 289, 167

Gruszecki, M., Murawski, K., \& Ofman, L. 2008, A\&A, 488, 757

Klimchuk, J. A., Antiochos, S. K., Norton, D., \& Watko, J. A. 2000, AAS, SPD meeting, 31

Korn, G., \& Korn, T. 1961, Mathematical Handbook for Scientists and Engineers (New York: McGraw-Hill)

Luna-Cardozo, M., Verth, G., \& Erdélyi, R. 2012, ApJ, 748, 110

Magyar, N., Van Doorsselaere, T., \& Marcu, A. 2015, A\&A, 582, A117

McEwan, M. P., Díaz, A. J., \& Roberts, B. 2008, A\&A, 481, 819

Morton, R. J., \& Erdélyi, R. 2009, ApJ, 707, 750

Morton, R. J., \& Erdélyi, R. 2010, A\&A, 519, A43

Nakariakov, V. M., Ofman, L., Deluca, E. E., Roberts, B., \& Davila, J. M. 1999, Science, 285,862

Ofman, L., \& Wang, T. J. 2008, A\&A, 482, L9

Ruderman, M. S. 2010, Sol. Phys., 267, 377

Ruderman, M. S. 2011a, A\&A, 534, A78

Ruderman, M. S. 2011b, Sol. Phys., 271, 41

Ruderman, M. S., \& Erdélyi, R. 2009, Space Sci. Rev., 149, 199

Ruderman, M. S., Verth, G., \& Erdélyi, R. 2008, ApJ, 686, 694

Ruderman, M. S., Petrukhin, N. S., \& Pelinovsky, E. 2016, Sol. Phys., 291, 1143

Ryutov, D. D., \& Ryutova, M. P. 1976, Sov. Phys. - JETP, 43, 491

Terradas, J., Arregui, I., Oliver, R., \& Ballester, J. L. 2008, ApJ, 678, L153

Terradas, J., Goossens, M., \& Ballai, I. 2010, A\&A, 515, A46

Tsuneta, S., Ichimoto, K., Katsukawa, Y., et al. 2008, ApJ, 688, 1374

Verth, G., \& Erdélyi, R. 2008, A\&A, 486, 1015

Watko, J. A., \& Klimchuk, J. A. 2000, Sol. Phys., 193, 77

Winebarger, A. R., DeLuca, E. E., \& Golub, L. 2001, ApJ, 553, L81

Winebarger, A. R., Warren, H., van Ballegooijen, A., DeLuca, E. E., \& Golub, L. 2002, ApJ, 567, L89 